\title{
Multicolour LEDs in educational demonstrations of physics and optometry
}

Paulis Paulins, Maris Ozolinsh

Paulis Paulins, Maris Ozolinsh, "Multicolour LEDs in educational demonstrations of physics and optometry," Proc. SPIE 9289, 12th Education and Training in Optics and Photonics Conference, 92891D (17 July 2014); doi: 10.1117/12.2070522

SPIE Event: 12th Education and Training in Optics and Photonics Conference, 2013, Porto, Portugal 


\title{
Multicolour LEDs in educational demonstrations of physics and optometry
}

\author{
Paulis Paulins*a ${ }^{\mathrm{a}}$, Maris Ozolinsh ${ }^{\mathrm{b}}$ \\ ${ }^{a}$ Dept. of Physics and Mathematics, University of Latvia, 8 Zellu Str., Riga, LATVIA LV1002; \\ ${ }^{\mathrm{b}}$ Inst.of Solid State Physics, University of Latvia, 8 Kengaraga Str., Riga, LATVIA LV1063
}

\begin{abstract}
LED light sources are used to design experimental setup for university courses teaching human color vision. The setup allows to demonstrate various vision characteristics and to apply for student practical exercises to study eye spectral sensitivity in different spectral range using heterochromatic flicker photometry. Technique can be used in laboratory works for students to acquire knowledge in visual perception, basics of electronics and measuring, or it can be applied as fully computer control experiment. Besides studies of the eye spectral sensitivity students can practice in trichromatic color matching and other visual perception tasks
\end{abstract}

Keywords: eye spectral sensitivity, heterochromatic flicker photometry, visual stimuli, LED, student laboratory work

\section{INTRODUCTION}

Human vision as one of sensory systems is of great interest for various professional fields. That is distinct, we get the most important information using our vision. Especially interesting is human ability to discriminate stimuli spectral characteristics - color vision [1]. It is studied by many well known people like physicist Newton, physiologist Hearing, poet and philosopher Goethe. The human color perception is of professional focus for painters, modern designers, engineers and many other practices. Students learn the color perception and human color vision in different university courses, such as psychology, physics or optometry. Physicians are interested in color vision deficiency, case when the eye photoreceptor layer lacks one or two type cone receptors and color discrimination is restricted, i.e. well known daltonism or other color „blindness”. Practical knowledge on diagnostics of color vision deficiency and on diversity of color blindness should be obtained in special university courses.

This paper focuses on implementation of practical expertise on trichromatic spectral characteristics of human eye. Students should acquire two main introductory tasks of color perception practically. The first is basics of color mixing. The second is determination of eye relative color sensitivity in regard to different perception spectra regions of cone receptors. Both tasks nowadays is very popular to accomplish using digital techniques - personal computers and monitors. Both of them are of special interest and significance. The forefront digital imaging solutions form special range of teaching interests. Availability of new light sources as narrow-spectrum light emitting diodes (LED) and portable diode pumped solid state (DPSS) lasers with easy electrical current control and relative fast OFF-ON-OFF switching transient changed spectrum of educational tools in universities. Application of LEDs avoids use of optical color filters that simplifies design of equipment. It allows to turn back to good analog lighting techniques and to build new convenient in use practical works in optics and optometry. The easiest way to determine eye relative spectral sensitivity is using of two techniques - heterochromatic flicker photometry and minimum motion illusion (apparent motion photometry) [2,3]. Minimum motion illusion is convenient to demonstrate better on PC screen due to complicity of LED stimulus design, and practical medical application designs using this effect to measure macular pigment optical density are quoted in literature [4]. However minimum motion illusion using LEDs is hard to adapt to provide students practical works.

Heterochromatic flicker photometry is based on finding the equiluminance of spectrally different light sources in eye. Here application of LEDs and DPSS laser sources simplifies the technical solution due to inertialess of sources in regard to transient times of eye physiology, availability of wide spectral choice of elements and no need of selective light filters. The present paper describes construction of light stimuli on LED basis, discusses the way of the stimuli application for heterochromatic flicker photometry technique in student practical works together with results of measurements, and touches perspective of DPSS laser light source use for similar experimental works.

*ozoma@latnet.lv 


\section{EXPERIMENTAL}

\subsection{Light emitting bars}

Students were provided with the set of two identical light bars with diameter $d=23 \mathrm{~mm}$ (Fig.1). Stimuli were turned from solid bars of PMMT. Surfaces of bars were finished to obtain smooth surface with diffuse emission of light after multireflections on the bar PMMA-air border. Bars were emitting the light of definite colour (in our case depending on set of LEDs mounted on both ends of the bars). Aluminium film glued on the back surfaces of bars was used to screen the bars' leak emission backward. Thus the working area of one stimuli was $A=23 \times 110 \mathrm{~mm}^{2}$. An additional aperture could be used to delimit working area. Two identical bars were used in case when spectral sensitivity was measured in symmetrically located peripheral zones of eye retina. A distant neutral background with ambient illumination or working computer screen was used as a neutral/color background.

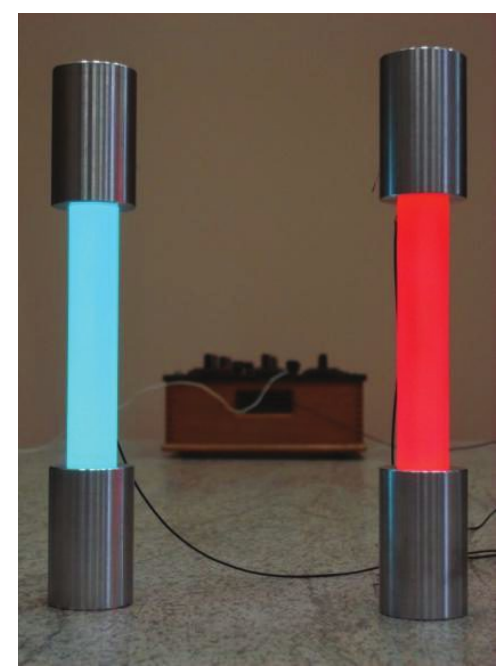

Figure 1. PMMA light bars with internal illumination of various color LED attached to both end surfaces of the bar. Working area $23 \times 110 \mathrm{~mm}$.

The corresponding bar CIE xyz colour coordinates were for blue, green, and red LED: $\mathrm{x}_{\mathrm{B}}=0.13, \mathrm{y}_{\mathrm{B}}=0.03, \mathrm{z}_{\mathrm{B}}=0.87$ (blue); $\mathrm{x}_{\mathrm{G}}=0.27, \mathrm{y}_{\mathrm{G}}=0.70, \mathrm{z}_{\mathrm{G}}=0.03$ (green); and $\mathrm{x}_{\mathrm{R}}=0.67, \mathrm{y}_{\mathrm{R}}=0.25, \mathrm{z}_{\mathrm{R}}=0.07$ (red). The colour coordinates and luminance were measured with Minolta CS-100A chronometer. LEDs used were of $1 \mathrm{~W}$ nominal power, and the light emission luminance $100 \mathrm{~cd} / \mathrm{m} 2$ for green stimuli was far from the maximum. Users were advised not to overrun these exploiting values to avoid heating and aging effects.

\subsection{Electronics}

LEDs were controlled using simple electronics components. The equipment was able to use standard computer parallel port PP output or logic gate TTL output to control for each light bar a pair of constant current source (CCS) electronics chips provided for LED consumers and available in market. The attached to opposite ends same color LEDs, i.e. red and green, were connected in series together with ballast resistors $\mathrm{R}_{\mathrm{BR}}$ and $\mathrm{R}_{\mathrm{BG}}$, To allow for students to change the LED current and hence the light emission, the variable resistor is connected parallel to the series circuit of LED. Using the magazines $R_{M R}$ and $R_{M G}$ instead of the variable resistors changes the LED current incrementally and simplifies the measurement data processing for students. As for heterochromatic flicker photometry LEDs of a comparable colors should be switched on alternatively, two LEDs are commuted by inverted and non-inverted output of the logic gate IC. Actual LED current values are measured by conventional multimeters, Often it is reasonable in student education activities to arrange measurements without computer control and data acquisition. In such case timer TIC plays the computer role, or the best case - the generator within the range 10-30 Hz performs triggering. 


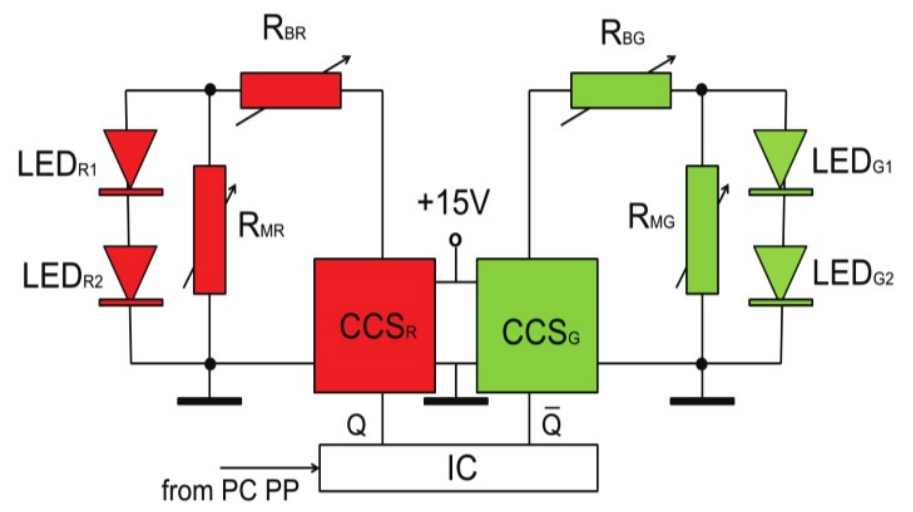

Figure 2. Schematics of electronic switching of pair of LED stimuli. $\mathrm{LED}_{\mathrm{R} 1}-\mathrm{LED}_{\mathrm{G} 2}$ red and green light emission diodes; $\mathrm{R}_{\mathrm{BR}}$ and $\mathrm{R}_{\mathrm{BG}}$ - ballast resistors; $\mathrm{R}_{\mathrm{MR}}$ and $\mathrm{R}_{\mathrm{MG}}$ - resistor magazines; $\mathrm{CCS}_{\mathrm{R}}$ and $\mathrm{CCS}_{\mathrm{G}}-$ constant current sources; IC- logical gate IC.

Same electronics can be adapted to control LED illumination in other school demonstrations, such as trichromatic color matching experiment. Here LED illumination is switched on continuously or quasi-continuously and three LED current is regulated either manually or using pulse-width modulation.

\section{PSYCHOPHYSICAL EXPERIMENTS AND DISCUSSION}

Human eye photoreceptor spectral sensitivity is depicted in Fig,3. The eye has rods that ensure vision in scotopic conditions and do not participate in color recognition. It has three color sensitive cone systems: S-cones that are short wavelength sensitive, middle wavelength sensitive M-cones, and long wavelength sensitive L-cones. The maximum of the eye spectral sensitivity $[\mathrm{dR} / \mathrm{d} \lambda](\lambda)$ - where $\mathrm{R}$ is the receptor response and $\lambda$ is wavelength, lies in spectrum range about $550 \mathrm{~nm}$. Some of people have either inherited or other lack of some cone system. The more often cases are protanopia - lack of L-cones and deuteranopia - lack of M-cones. Then the eye spectral sensitivity curve is deformed, and also color discrimination is limited. Besides the color deficient observer perceives some of rainbow color as colorless gray light.

The human relative eye spectral sensitivity can be measured using heterochromatic flicker photometry. The technique principle lays in the fact that alternatively changing of visual stimuli induces flickering sense response in case when stimuli are not equiluminant [2]. To reveal flickering the optimum frequency of periodic switching depends on the stimuli intensity, the color used as stimuli and on the adaptation to ambient illumination. Besides the physical factors the

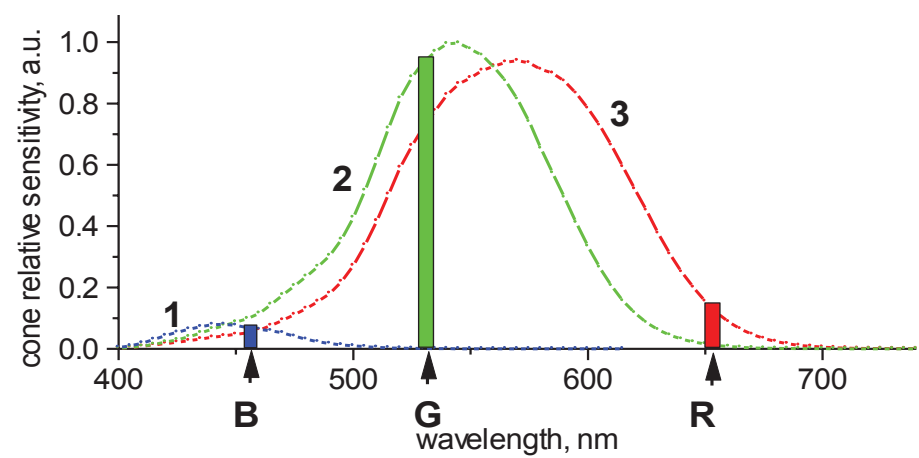

Figure 3. Spectral characteristics of human eye cone photoreceptors: short wavelength sensitive $-\mathrm{S}$ cones (curve 1); middle wavelength sensitive - $\mathrm{M}$ cones (curve 1); long wavelength sensitive - $\mathrm{L}$ cones (curve 3 ). Arrows show spectral emission wavelength of blue B, green G, and red R light diodes. 


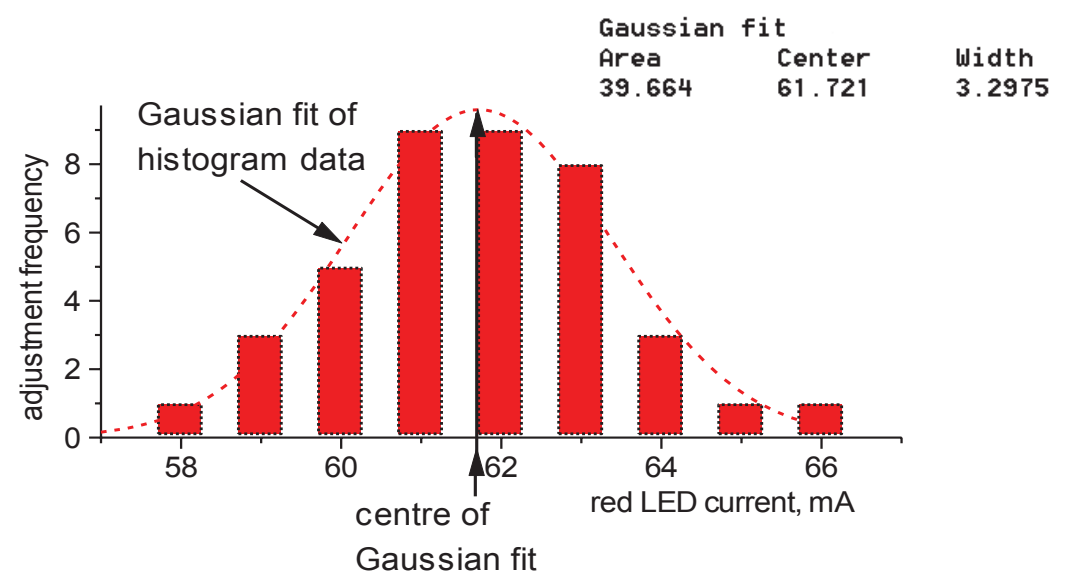

Figure 4. Histogram of measurement series to determine the condition of equiluminance of the red and green LED stimuli for eye central vision using heterochromatic flicker photometry. Flicker frequency equals to $15 \mathrm{~Hz}$. Horizontal axis scaled for red LED current. Opposite green stimuli LED current constant $\mathrm{I}_{\text {LEDgreen }}=15 \mathrm{~mA}$.

eye relative sensitivity depends also on eccentricity of excited photoreceptors, on the presence and optical thickness of eye macula pigment layer and other factors of interest for ophthalmologists and optometrists.

Students place the rod stimulus in front of eyes in a fixed distance. One color LED (i.e., green) current is kept constant. Other color LED (i.e., red) current is adjusting. If the red stimulus luminance for the individual observer is lower then the green stimulus luminance the student observes distinct flicker effect, if the flicker frequency $f$ is lower as the eye critical flicker fusion frequency $(f>50 \mathrm{~Hz})$. Adjusting LED current minimizes the stimulus flickering sense. If no flickering is observed in a wider range of current adjusting, or flickering is minimized but does not diminish, the stimulus color changing frequency can be altered: increased in the first case or decreased in the latter case. The LED current for running adjustment is documented. To obtain statistically significant and comparable results adjusting can be repeated 30-40 times. Luminance calibration in regard to standard observer is done by Minolta CS-100A chronometer.

Fig. 4 shows results of one measurement series to find the red and green LED equiluminance condition for an individual observer. Fitting the distribution frequency with standard Gaussian procedure one finds the LED current average value at equiluminance and standard deviation SD of LED current adjustment.

Performing the experiment preparatory work, carrying out adjustment series for different color stimuli equiluminance determination, processing statistically results and their analysis student acquires knowledge about the nature of the visual perception and improves their expertise in electronics and measurements.

Used in experimental setup light sources are of relative high color purity and can be successfully used also in color matching tasks. In applications for some specific measurements of visual perception the setup needs selection of spectral emission characteristics of sources, i.e. narrow spectrum LED sources in blue range with small [5] mutual spectral shift or laser line emission at all. For such tasks use of miniature DPSS laser diodes is very optimal. Electronics needed for DPSS laser diodes emission computer control is similar to that used in the present paper. Visual stimuli can be designed using of side emitting optical fibers [6]. DPSS lasers and low-cost opto-mechanics elements of emission and fibre matching for student research work are available [7] via internet.

\section{CONCLUSIONS}

Easy intensity controllable LEDs allow to design various experimental setups for use in optics, optometry and other university teaching courses. Paper focuses on design of visual color stimuli and the measurement technique of heterochromatic flicker photometry to determine relative subjective eye sensitivity in different spectral regions. Technique can be modified and applied in wider interest areas using other narrow emission spectrum sources such as portable DPSS lasers. 


\section{ACKNOWLEDGEMENT}

Author M. Ozolinsh is supported by project ERAF 2010/0259/2DP/2.1.1.1.0/10/APIA/VIAA/137.

\section{REFERENCES}

[1] Gegenfurtner, K.R. and Sharpe, L.T., [Color Vision: From Genes to Perception], Cambridge University Press, Cambridge, 492p. (2001).

[2] Pokorny, J., Smith, V. and Lutze, M., "Heterochromatic modulation photometry," J. Opt. Soc. Amer. A6, 1618-1623 (1989).

[3] Moreland, J.D., "Macular pigment assessment by motion photometry," Arch. Biochem. Biophys. 430, 143-148 (2004).

[4] Bartlett, H., Acton, J. and Eperjesi, F., "Clinical evaluation of the MacuScope macular pigment densitometer," Br. J. Ophthalmol. 92, 328-331 (2010).

[5] Ozolinsh, M., and Paulins, P., "LED based dual wavelength heterochromatic flicker method for separate evaluation of lutein and zeaxanthin in retina," IFMBE Proc. 38, 51-54 (2013).

[6] Spigulis, J., "Side-emitting fibers brighten our world," OPN October 2005, 35-39.

[7] <http://www.aixiz.com/> 